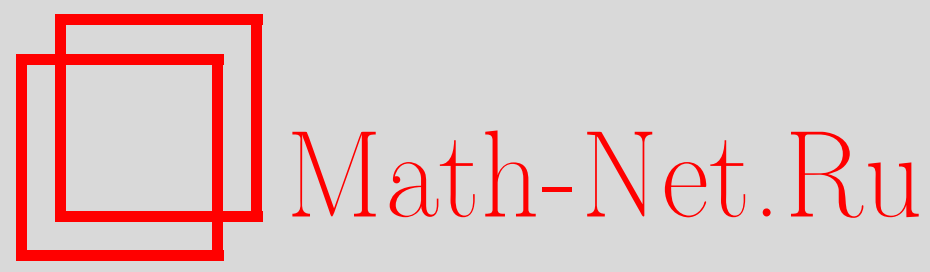

Д. А. Яроцкий, Центральная предельная теорема для одного класса неоднородных случайных блужданий, Матем. заметки, 2001, том 69, выпуск 5, 751-757

DOI: https://doi.org/10.4213/mzm538

Использование Общероссийского математического портала Math-Net.Ru подразумевает, что вы прочитали и согласны с пользовательским соглашением http://www . mathnet.ru/rus/agreement

Параметры загрузки:

IP : 52.6 .47 .48

26 апреля 2023 г., 13:07:40

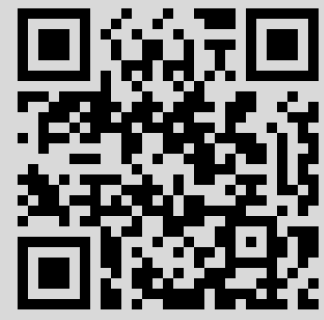




\section{ЦЕНТРАЛЬНАЯ ПРЕДЕЛЬНАЯ ТЕОРЕМА ДЛЯ ОДНОГО КЛАССА НЕОДНОРОДНЫХ СЛУЧАЙНЫХ БЛУЖДАНИЙ}

\section{Д. А. Яроцкий}

Рассматривается неоднородное по пространству случайное блуждание $\eta_{t}$ на решетке $\mathbb{Z}^{\nu}=\mathbb{Z}^{m} \times \mathbb{Z}^{n}$. Пусть $\eta_{t}^{0}-$ некоторое однородное (по времени и пространству) случайное блуждание, и $\eta_{t}$ получено из него посредством изменения переходных вероятностей на множестве $A=\bar{A} \times \mathbb{Z}^{n},|\bar{A}|<\infty$, так что блуждание остается однородным только по отношению к подгруппе $\mathbb{Z}^{n}$ группы $\mathbb{Z}^{\nu}$. Показано, что если $m \geqslant 2$ или снос отличен от нуля, то для $\eta_{t}$ выполняется центральная предельная теорема.

Библиография: 5 названий.

1. Введение. Рассмотрим марковское случайное блуждание на решетке $\mathbb{Z}^{\nu}$ с вероятностями перехода

$$
\operatorname{Pr}_{0}(x \rightarrow y):=p(y-x),
$$

где $p(\cdot)$ - функция, определяющая распределение вероятностей на $\mathbb{Z}^{\nu}$ :

$$
p(x) \geqslant 0, \quad \sum_{x \in \mathbb{Z}^{\nu}} p(x)=1 .
$$

Предположим, что функция $p(\cdot)$ финитна в том смысле, что $p(x)=0$, если $\|x\|$ достаточно велика.

Пусть $\eta_{t}^{0}, t=0,1,2, \ldots,-$ случайное блуждание, начинающееся из 0 и обладающее переходньми вероятностями (1):

$$
\begin{gathered}
\mathrm{P}\left\{\eta_{0}^{0}=0\right\}=1 \\
\mathrm{P}\left\{\eta_{t+1}^{0}=y \mid \eta_{t}^{0}=x\right\}=\operatorname{Pr}_{0}(x \rightarrow y) .
\end{gathered}
$$

Определим $\nu$-мерньй вектор $b=\left(b_{i}\right)$ и самосопряженную неотрицательную $(\nu \times \nu)$-матрицу $\left(a_{i j}\right)$ равенствами

$$
\begin{gathered}
b_{i}=\sum_{x \in \mathbb{Z}^{\nu}} x_{i} p(x), \\
a_{i j}=\sum_{x \in \mathbb{Z}^{\nu}}\left(x_{i}-b_{i}\right)\left(x_{j}-b_{j}\right) p(x),
\end{gathered}
$$


где $x_{i}$ обозначает $i$-ю координату. Тогда, как следует из центральной предельной теоремы, распределение $\left(\eta_{t}^{0}-t b\right) / \sqrt{t}$ слабо сходится к гауссовскому со средним 0 и матрищей ковариаций $\left(a_{i j}\right)$ при $t \rightarrow \infty$.

Пусть $\nu=m+n$ где $m, n \in \mathbb{Z}, m \geqslant 0, n \geqslant 1$. В этом случае $\mathbb{Z}^{\nu}=\mathbb{Z}^{m} \times \mathbb{Z}^{n}$, и если $x \in \mathbb{Z}^{\nu}$, то обозначим $x=(\bar{x}, \overline{\bar{x}})$, где $\bar{x} \in \mathbb{Z}^{m}, \overline{\bar{x}} \in \mathbb{Z}^{n}$.

Теперь рассмотрим неоднородное случайное блуждание на $\mathbb{Z}^{\nu}$ с вероятностями перехода вида

$$
\operatorname{Pr}(x \rightarrow y):=p(y-x)+v(\bar{x}, \bar{y}, \overline{\bar{y}}-\overline{\bar{x}}),
$$

где первое слагаемое в правой части то же самое, что и в (1), а второе рассматривается как возмущение. Конечно, мы предполагаем, что функция $v(\cdot, \cdot, \cdot)$ такова, что для любого $x$ правая часть (3) определяет распределение вероятностей

$$
\begin{gathered}
p(y-x)+v(\bar{x}, \bar{y}, \overline{\bar{y}}-\overline{\bar{x}}) \geqslant 0 \\
\sum_{y \in \mathbb{Z}^{\nu}}(p(y-x)+v(\bar{x}, \bar{y}, \overline{\bar{y}}-\overline{\bar{x}}))=1 .
\end{gathered}
$$

Вид второго слагаемого в правой части (3) показывает, что возмущение сохраняет однородность по отношению к транслящиям, соответствующим подрешетке $\mathbb{Z}^{n}$.

Пусть функция $v$ также финитна. В этом случае "область действия возмущения"

$$
A:=\left\{x \in \mathbb{Z}^{\nu} \mid \exists y v(\bar{x}, \bar{y}, \overline{\bar{y}}-\overline{\bar{x}}) \neq 0\right\}
$$

является цилиндрическим множеством:

$$
A=\bar{A} \times \mathbb{Z}^{n}
$$

где $\bar{A}$ - некоторое конечное подмножество $\mathbb{Z}^{m}$.

Пусть $\eta_{t}, t=0,1,2 \ldots$, - выходящее из 0 неоднородное случайное блуждание с переходными вероятностями, определенньми формулой (3).

В настоящей работе при некоторых дополнительных предположениях будет доказан следующий результат.

ТЕоремА. Пусть $b_{i}$ и $a_{i j}$ определень формулами $\left(2^{\prime}\right),\left(2^{\prime \prime}\right)$. Если $b \neq 0$ или $n \geqslant 2$, то распределение $\left(\eta_{t}-t b\right) / \sqrt{t}$ слабо сходится $\kappa$ гауссовскому со средним 0 u матричей ковариачий $\left(a_{i j}\right)$ при $t \rightarrow \infty$.

Заметим, что этот результат не имеет места, если $b=0$ и $n=1$. Оказьвается, что в этом случае предельное распределение существует, но отлично от гауссовского (см. [1]).

Перед тем как сформулировать дополнительные предположения, введем некоторые обозначения.

Пусть $\eta_{t}=\left(\bar{\eta}_{t}, \overline{\bar{\eta}}_{t}\right)$, где случайные процессы $\bar{\eta}_{t} \in \mathbb{Z}^{m}, \overline{\bar{\eta}}_{t} \in \mathbb{Z}^{n}$ являются проекциями случайного процесса $\eta_{t}$. Легко видеть из $(3)$, что $\bar{\eta}_{t}$ также будет марковским процессом. Обозначая его переходные вероятности через $\overline{\operatorname{Pr}}(\bar{x} \rightarrow \bar{y})$, имеем

$$
\overline{\operatorname{Pr}}(\bar{x} \rightarrow \bar{y})=\sum_{\overline{\bar{y}} \in \mathbb{Z}^{n}} \operatorname{Pr}((\bar{x}, \overline{\bar{x}}) \rightarrow(\bar{y}, \overline{\bar{y}})),
$$


где $\overline{\bar{x}}$ произвольно.

Аналогично мы вводим переходные вероятности для $\bar{\eta}_{t}^{0} \in \mathbb{Z}^{m}$ :

$$
\overline{\operatorname{Pr}}_{0}(\bar{x} \rightarrow \bar{y})=\sum_{\bar{y} \in \mathbb{Z}^{n}} \operatorname{Pr}_{0}((\bar{x}, \overline{\bar{x}}) \rightarrow(\bar{y}, \overline{\bar{y}}))=\bar{p}(\bar{y}-\bar{x})
$$

где

$$
\bar{p}(\bar{x}):=\sum_{\overline{\bar{x}} \in \mathbb{Z}^{n}} p(\bar{x}, \overline{\bar{x}})
$$

Мы будем предполагать, что вьполнены следующие два условия.

1. Назовем непустое конечное подмножество $\bar{B}$ решетки $\mathbb{Z}^{m}$ ловушкой, если для любых $\bar{x} \in \bar{B}$ и $\bar{y} \notin \bar{B}$ выполнено $\overline{\operatorname{Pr}}(\bar{x} \rightarrow \bar{y})=0$. Мы будем считать, что у рассматриваемого блуждания нет ловушек.

2. Мы будем считать, что однородное блуждание $\bar{\eta}_{t}^{0}$ неприводимо в следующем смысле:

$$
\left\{\sum a_{k} \bar{x}_{k} \mid a_{k} \in \mathbb{Z}, \bar{x}_{k} \in \operatorname{supp} \bar{p}\right\}=\mathbb{Z}^{m}
$$

Теорема обобщает некоторые результаты работ [1], [2].

2. Доказательство теоремы. Достаточно показать, что

$$
\Delta_{f}(t):=\mathrm{E}\left(f\left(\frac{\eta_{t}^{0}-t b}{\sqrt{t}}\right)\right)-\mathrm{E}\left(f\left(\frac{\eta_{t}-t b}{\sqrt{t}}\right)\right) \rightarrow 0
$$

при $t \rightarrow \infty$ для любой достаточно гладкой финитной $f: \mathbb{R}^{\nu} \rightarrow \mathbb{R}$. Рассмотрим функцию $q(t)$

$$
q(t):=\sum_{\tau=0}^{t-1} \mathrm{P}\left\{\eta_{t} \in A\right\}
$$

где $A$ определено формулой (4).

ЛЕмма 1. Существует константа $C>0$ такая, что для всех $t$

$$
\left|\Delta_{f}(t)\right|<\frac{C}{\sqrt{t}} q(t)\|\nabla f\|
$$

$2 \partial e$

$$
\|\nabla f\|:=\max _{\mathbb{R}^{\nu}}\left(\sum_{i=1}^{\nu}\left|\frac{\partial f}{\partial x_{i}}\right|^{2}\right)^{1 / 2} .
$$


ДокАЗАТЕЛЬСТво. Пусть

$$
C=\max _{x \in A}\left(\sum_{y \in \mathbb{Z}^{\nu}}\|y-x\| \operatorname{Pr}_{0}(x \rightarrow y)+\sum_{y \in \mathbb{Z}^{\nu}}\|y-x\| \operatorname{Pr}(x \rightarrow y)\right),
$$

где $\|y-x\|:=\left(\sum_{i=1}^{\nu}\left(y_{i}-x_{i}\right)^{2}\right)^{1 / 2}$.

Достаточно показать, что для любой финитной гладкой $g: \mathbb{R}^{\nu} \rightarrow \mathbb{R}$ и для любого $t=1,2 \ldots$

$$
\left|\mathrm{E}\left(g\left(\eta_{t}^{0}\right)\right)-\mathrm{E}\left(g\left(\eta_{t}\right)\right)\right|<C q(t)\|\nabla g\| .
$$

Воспользуемся индукцией. Пусть это утверждение вьполнено для $t=t_{0}$. Тогда для $t=t_{0}+1$ имеем

$$
\begin{aligned}
\mid \mathrm{E} & \left(g\left(\eta_{t_{0}+1}^{0}\right)\right)-\mathrm{E}\left(g\left(\eta_{t_{0}+1}\right)\right) \mid \\
= & \left|\sum_{x, y \in \mathbb{Z}^{\nu}} \mathrm{P}\left\{\eta_{t_{0}}^{0}=x\right\} \operatorname{Pr}_{0}(x \rightarrow y) g(y)-\sum_{x, y \in \mathbb{Z}^{\nu}} \mathrm{P}\left\{\eta_{t_{0}}=x\right\} \operatorname{Pr}(x \rightarrow y) g(y)\right| \\
\leqslant & \left|\sum_{x, y \in \mathbb{Z}^{\nu}}\left(\mathrm{P}\left\{\eta_{t_{0}}^{0}=x\right\}-\mathrm{P}\left\{\eta_{t_{0}}=x\right\}\right) \operatorname{Pr}_{0}(x \rightarrow y) g(y)\right| \\
& +\left|\sum_{x, y \in \mathbb{Z}^{\nu}} \mathrm{P}\left\{\eta_{t_{0}}=x\right\}\left(\operatorname{Pr}_{0}(x \rightarrow y)-\operatorname{Pr}(x \rightarrow y)\right) g(y)\right| \\
\leqslant & \left|\sum_{x, z \in \mathbb{Z}^{\nu}}\left(\mathrm{P}\left\{\eta_{t_{0}}^{0}=x\right\}-\mathrm{P}\left\{\eta_{t_{0}}=x\right\}\right) p(z) g(x+z)\right| \\
& +\sum_{x \in A} \mathrm{P}\left\{\eta_{t_{0}}=x\right\}\left(\left|\sum_{y \in \mathbb{Z}^{\nu}} \operatorname{Pr}_{0}(x \rightarrow y) g(y)-g(x)\right|+\left|\sum_{y \in \mathbb{Z}^{\nu}} \operatorname{Pr}(x \rightarrow y) g(y)-g(x)\right|\right) \\
\leqslant & \sum_{z \in \mathbb{Z}^{\nu}} p(z)\left|\sum_{x \in \mathbb{Z}^{\nu}}\left(\mathrm{P}\left\{\eta_{t_{0}}^{0}=x\right\}-\mathrm{P}\left\{\eta_{t_{0}}=x\right\}\right) g(x+z)\right| \\
& +\sum_{x \in A} \mathrm{P}\left\{\eta_{t_{0}}=x\right\} C\|\nabla g\| \\
\leqslant & \sum_{z \in \mathbb{Z}^{\nu}} p(z) C q\left(t_{0}\right)\|\nabla g\|+\sum_{x \in A} \mathrm{P}\left\{\eta_{t_{0}}=x\right\} C\|\nabla g\| \\
= & C q\left(t_{0}+1\right)\|\nabla g\| .
\end{aligned}
$$

Мы использовали то, что

$$
\left|\sum_{y \in \mathbb{Z}^{\nu}} \operatorname{Pr}_{0}(x \rightarrow y) g(y)-g(x)\right|+\left|\sum_{y \in \mathbb{Z}^{\nu}} \operatorname{Pr}(x \rightarrow y) g(y)-g(x)\right| \leqslant C\|\nabla g\|,
$$

а также то, что $\|\nabla g(\cdot+z)\|=\|\nabla g(\cdot)\|$. Основание индукции $t=1$ проверяется аналогично. Лемма доказана.

Теперь нашей целью будет рассмотреть функцию $q(t)$ и доказать, что

$$
q(t)=o(\sqrt{t})
$$


так как благодаря лемме 1 из этой оценки следует утверждение теоремы.

Рассмотрим стохастический оператор $\mathscr{T}$, определенный на множестве функций $\{u$ : $\left.\mathbb{Z}^{\nu} \rightarrow \mathbb{R}\right\}$ следующим образом:

$$
\mathscr{T} u(x):=\sum_{y \in \mathbb{Z}^{\nu}} u(y) \operatorname{Pr}(x \rightarrow y) .
$$

Пусть функция $w$ обладает таким свойством:

$$
(\mathscr{T}-\mathscr{I}) w=\chi_{A},
$$

где $\chi_{A}$ обозначает индикатор множества $A$ и $\mathscr{I}$ есть единичньй оператор. Тогда

$$
\mathscr{T}^{t} w(0)=\mathscr{T}^{t-1} w(0)+\mathscr{T}^{t-1} \chi_{A}(0)=\cdots=\sum_{\tau=0}^{t-1} \mathscr{T}^{\tau} \chi_{A}(0)=q(t)+w(0) .
$$

Мы будем строить функцию $w$, удовлетворяющую (8) и для которой

$$
\mathscr{T}^{t} w(0)=o(\sqrt{t})
$$

так как благодаря (9) из существования такой функции следует оценка (7).

Пусть $\overline{\mathscr{T}}_{0}$ и $\overline{\mathscr{T}}$ - стохастические операторы, определенные на множестве $\left\{\bar{u}: \mathbb{Z}{ }^{m} \rightarrow \mathbb{R}\right\}$ следующим образом:

$$
\overline{\mathscr{T}}_{0} \bar{u}(\bar{x}):=\sum_{\bar{y} \in \mathbb{Z}^{m}} \bar{u}(\bar{y}) \overline{\operatorname{Pr}}_{0}(\bar{x} \rightarrow \bar{y}), \quad \overline{\mathscr{T}} \bar{u}(\bar{x}):=\sum_{\bar{y} \in \mathbb{Z}^{m}} \bar{u}(\bar{y}) \overline{\operatorname{Pr}}(\bar{x} \rightarrow \bar{y}) .
$$

Пусть для функции $\bar{w}$ вьполнено условие

$$
(\overline{\mathscr{T}}-\mathscr{I}) \bar{w}=\chi_{\bar{A}},
$$

где $\mathscr{I}$ - единичньй оператор и $\chi_{\bar{A}}-$ индикатор множества $\bar{A}$, определенного в $(5)$. Тогда функция $w: \mathbb{Z}^{\nu} \rightarrow \mathbb{R}$, заданная формулой

$$
w((\bar{x}, \overline{\bar{x}})):=\bar{w}(\bar{x})
$$

удовлетворяет условию (8). Более того, $\mathscr{T}^{t} w(0)=\overline{\mathscr{T}}^{t} \bar{w}(0)$ и (10) эквивалентно

$$
\overline{\mathscr{T}}^{t} \bar{w}(0)=o(\sqrt{t}) \text {. }
$$

Таким образом, задача сведена к нахождению решения (11), удовлетворяющего (12).

Сначала рассмотрим следующую аналогичную задачу для стохастического оператора $\overline{\mathscr{T}}_{0}$ :

$$
\left(\overline{\mathscr{T}}_{0}-\mathscr{I}\right) \varphi=\delta
$$

где $\delta(\bar{x})=\chi_{\{\bar{x}=0\}}$. Заметим, что согласно $(6)$ подматрица $\overline{\mathscr{A}}:=\left(a_{i j}\right)_{i, j=1}^{m}$ матрицы $\left(a_{i j}\right)_{i, j=1}^{\nu}$ невырождена, и введем новую норму $\|\cdot\|_{1}$ в $\mathbb{R}^{m}$

$$
\|\bar{x}\|_{1}^{2}:=\left(\overline{\mathscr{A}}^{-1} \bar{x}, \bar{x}\right),
$$

где $(\bar{x}, \bar{y}):=\sum_{i=1}^{m} \bar{x}_{i} \bar{y}_{i}$.

Обозначим через $\mathscr{F} \bar{p}$ преобразование $Ф$ урье от $\bar{p}$

$$
\mathscr{F} \bar{p}(\theta):=\sum_{\bar{x} \in \mathbb{Z}^{m}} \bar{p}(\bar{x}) \exp \{i(\theta, \bar{x})\},
$$

где $\theta \in T^{m}:=[-\pi, \pi]^{m}$. Согласно $(6) \mathscr{F} \bar{p}(\theta)=1$ тогда и только тогда, когда $\theta=0$ (см. [3]). 
Лемма 2. Уравнение (13) обладает решением $\varphi$ таким, что

a) если $m \geqslant 3$, mo $\varphi(\bar{x}) \rightarrow 0$ npu $\bar{x} \rightarrow \infty$;

b) если $m=2 u \bar{b}:=\left(b_{i}\right)_{i=1}^{m} \neq 0$, mo maкже $\varphi(\bar{x}) \rightarrow 0$ nрu $\bar{x} \rightarrow \infty$;

c) если $m=2 u \bar{b}=0$, mo

$$
\varphi(\bar{x})=\frac{(\operatorname{det} \overline{\mathscr{A}})^{-1 / 2}}{\pi} \ln \|\bar{x}\|_{1}+o(1) ;
$$

d) если $m=1$ u $\bar{b} \neq 0$, то существуют предель $\varphi(\bar{x}) \rightarrow \varphi_{ \pm}$при $\bar{x} \rightarrow \pm \infty$.

ДокАЗАТЕЛЬСтво. Для $\varphi$ имеются явные формулы (см. [4]): в случаях а) и b)

$$
\varphi(\bar{x})=\frac{1}{(2 \pi)^{m}} \int_{T^{m}} \frac{\exp \{i(\bar{x}, \theta)\}}{\mathscr{F} \bar{p}(\theta)-1} d \theta,
$$

а в случаях с) и d)

$$
\varphi(\bar{x})=\frac{1}{(2 \pi)^{m}} \int_{T^{m}} \frac{\exp \{i(\bar{x}, \theta)\}-1}{\mathscr{F} \bar{p}(\theta)-1} d \theta .
$$

Тогда утверждения а) и b) являются следствиями леммы Римана-Лебега, так как, как легко проверить, функция $(\mathscr{F} \bar{p}-1)^{-1}$ интегрируема в этих двух случаях.

Мы не будем приводить вывод с) и d); заметим лишш, что примеры доказательств подобных оценок можно найти в [4], [5].

Теперь вернемся к решению уравнения (11) (для неоднородного блуждания). Мы построим решение этого уравнения в виде линейной комбинации найденных вьше функций $\varphi$, решающих уравнение (13) (для однородного блуждания). А именно, пусть

$$
\bar{w}(\bar{x}):=\alpha_{1} \varphi\left(\bar{x}-\bar{x}^{(1)}\right)+\cdots+\alpha_{k} \varphi\left(\bar{x}-\bar{x}^{(k)}\right),
$$

где $\bar{x}^{(1)}, \ldots, \bar{x}^{(k)}$ - это все точки множества $\bar{A}$. Тогда, как легко увидеть из определения $\bar{A},(\overline{\mathscr{T}}-\mathscr{I}) \bar{w}(\bar{x})=\beta_{1} \delta\left(\bar{x}-\bar{x}^{(1)}\right)+\cdots+\beta_{k} \delta\left(\bar{x}-\bar{x}^{(k)}\right)$, что равно $\chi_{\bar{A}}$, если все $\beta_{s}=1$. Постоянные $\beta_{s}$ линейно зависят от постоянных $\alpha_{s}$, и если мы докажем, что ядро отображения $\left\{\alpha_{s}\right\} \mapsto\left\{\beta_{s}\right\}$ тривиально, то, пользуясь хорошо известной теоремой линейной алгебры, мы можем сказать, что существует набор $\alpha_{s}$, для которого $\beta_{s} \equiv 1$.

Предположим, что набор $\alpha_{s}$ принадлежит ядру, т.е. $(\overline{\mathscr{T}}-\mathscr{I}) \bar{w} \equiv 0$.

Пусть $\bar{x}$ - точка, в которой функция $\bar{w}$, полученная по формуле $(14)$, достигает экстремума (существование такой точки нетрудно вьвести из оценок леммы 2).

Легко видеть, что в этом случае если $\overline{\operatorname{Pr}}(\bar{x} \rightarrow \bar{y}) \neq 0$ для некоторого $\bar{y}$, то $\bar{w}(\bar{y})=\bar{w}(\bar{x})$, так как функция $\overline{\operatorname{Pr}}(\bar{x} \rightarrow \cdot)$ определяет распределение вероятностей.

Отсюда следует, что для лишенного ловушек случайного блуждания $\bar{w} \equiv$ const. $\mathrm{B}$ частности, $\alpha_{s}=\left(\overline{\mathscr{T}}_{0}-\mathscr{I}\right) \bar{w}\left(\bar{x}^{(s)}\right)=0$ для всех $s$.

Итак, найдется набор $\alpha_{s}$, для которого $\beta_{s} \equiv 1$ и соответствующее $\bar{w}$ удовлетворяет (11). Остается показать, что условие (12) также вьполнено. Действительно, если $m \geqslant 3$ или $\bar{b} \neq 0$, то согласно лемме 2 последовательность $\overline{\mathscr{T}}^{t} \bar{w}(0)$ ограничена. Если же $m=2$ и $\bar{b}=0$, то, как легко следует из финитности функций $p, v$ и леммы 2 , $\left|\overline{\mathscr{T}}^{t} \bar{w}(0)\right| \leqslant c_{1}+c_{2} \ln t$, где $c_{1}, c_{2}$ - некоторые константы.

Теорема доказана.

Автор выражает благодарность Е. А. Жижиной и Р. А. Минлосу за помощь в работе и полезное обсуждение результатов. 


\section{СПИСОК ЦИТИРОВАННОЙ ЛИТЕРАТУРЫ}

[1] ЖКижина Е. А., Минлос Р. А. Локальная предельная теорема для неоднородного случайного блуждания на решетке. // Теория вероятностей и ее применения. 1994. Т. 39. № 3. C. $513-529$.

[2] Minlos P. A., Zhizhina E. A. The limiting theorems for a random walk of two particles on the lattice $\mathbb{Z}^{\nu} / /$ Potential Analysis. 1996. V. 5. P. 139-172.

[3] Гихман И. И., Скороход А. В. Теория случайных процессов. Т. 1. М.: Наука, 1971.

[4] Спицер Ф. Принципы случайного блуждания. М.: Мир, 1969.

[5] Duffin R. J. Discrete potential theory // Duke Math. J. 1953. V. 20. P. 233-251.

Московский государственный университет им. М.В. Ломоносова

Поступило

E-mail: yarotsky@mail.ru

Исправленный вариант

05.04 .2000 